\title{
Structural Brain MR Imaging Changes Associated with Obsessive-Compulsive Disorder in Patients with Multiple Sclerosis
}

\author{
E. Tinelli, A. Francia, E.M. Quartuccio, M. Morreale, G.M. Contessa, S. Pascucci, E. Sbardella, C. Pozzilli, and P. Pantano
}

\begin{abstract}
BACKGROUND AND PURPOSE: Psychiatric symptoms occur in approximately 30\% of patients with MS. Such symptoms include OCD, which may interfere heavily with the patient's daily life. We hypothesized that the widespread involvement of both GM and WM, which characterizes MS, may be responsible for the occurrence of OCD when specific brain structures are affected. The aim of this study was to evaluate the relationship between GM and WM tissue damage and OCD in patients with MS.
\end{abstract}

MATERIALS AND METHODS: We evaluated 16 patients with relapsing-remitting MS who had been diagnosed with OCD on the basis of the Diagnostic and Statistical Manual of Mental Disorders (4th edition) and 15 age- and sex-matched patients with relapsing-remitting MS with no psychiatric disorders as a CG. The MR study (1.5T) included 3D T1-weighted fast-field echo sequences, DTI (32 directions), and conventional MRI. Images were processed using SPM5, FSL, and Jim 5.0 software to evaluate VBM, TBSS, and global and regional LV, respectively.

RESULTS: The VBM analysis revealed a set of clusters of reduced GM volume in the OCD group, compared with the CG, located in the right inferior and middle temporal gyri and in the inferior frontal gyrus. TBSS did not detect any differences in the FA values between the 2 groups; global and regional LV values also did not differ significantly between the 2 groups.

CONCLUSIONS: Our study suggests that OCD in MS may be caused by damage in the right frontotemporal cortex.

ABBREVIATIONS: $C G=$ control group; EDSS = Expanded Disability Status Scale; FA = fractional anisotropy; GM = gray matter; $L V=$ T2 lesion volume; OCD = obsessive-compulsive disorder; TBSS = tract-based spatial statistics; VBM = voxel-based morphometry; Y-BOCS = Yale-Brown Obsessive Compulsive Scales

M $\mathrm{S}$ is the most common chronic inflammatory and degenerative disease of the central nervous system. Besides neurologic signs and symptoms, MS may be associated with various psychiatric manifestations such as depression, anxiety, euphoria, emotional lability, mania, and psychosis. ${ }^{1-3}$ It has been estimated that approximately $30 \%$ of patients with MS experience psychiatric disorders during the disease course. ${ }^{1}$ Investigations of psychiatric disturbances in MS have usually focused on depression and anxiety ${ }^{3}$ or psychosis. ${ }^{4}$ The lifetime rate of anxiety disorders in the MS population was reported by the National Co-

Received February 15, 2012; accepted after revision May 8.

From the Departments of Neurology and Psychiatry (E.T., A.F., E.M.Q., S.P., C.P., P.P.), Medico-Surgical Sciences and Biotechnologies-Section of Neurology (M.M.), and Psychology (E.S.), Sapienza University of Rome, Rome, Italy; and Department of Occupational Hygiene (G.M.C.), Italian Workers Compensation Authority, Rome, Italy.

This work was supported in part by a grant from the Federazione Italiana Sclerosi Multipla (FISM) 2010/R/26.

Please address correspondence to Emanuele Tinelli, MD, Department of Neurology and Psychiatry, Sapienza University of Rome, Viale dell'Universitá 30, 00185 Rome, Italy; e-mail: ematine@libero.it

http://dx.doi.org/10.3174/ajnr.A3210 morbidity Survey as 25\%. ${ }^{5}$ More recently, Korostil and Feinstein ${ }^{6}$ found that $35.7 \%$ of their sample of 140 patients with MS had an anxiety disorder at some time during their lifetime, attributable to one of the following 3 disorders: generalized anxiety disorder, OCD, and panic disorder. In particular, the lifetime prevalence of OCD in MS was $8.6 \%$ compared with $2.5 \%$ in the general population. $^{6}$

OCD is characterized by distressing repetitive thoughts, impulses, or images that are intense, frightening, absurd, or unusual. These thoughts are followed by ritualized actions that are usually bizarre and irrational. These ritual actions, known as compulsions, help reduce anxiety caused by one's obsessive thoughts. Often described as the "disease of doubt," the sufferer usually knows the obsessive thoughts and compulsions are irrational but, on another level, fears they may be true. Therefore, OCD causes marked distress and may heavily interfere with daily life and social relationships.

In primary OCD (ie, not associated with other neurologic diseases), neuroimaging studies have revealed structural and/or functional abnormalities in specific brain structures, particularly 
in the fronto-striato-thalamic circuitry, ${ }^{7-9}$ which points to an organic etiology of this psychiatric disease.

We hypothesized that the widespread involvement of both GM and WM, which characterizes MS, may be responsible for the occurrence of OCD when specific brain structures are affected. The aim of this study was to evaluate the relationship between GM and WM tissue damage and OCD in patients with MS.

\section{MATERIALS AND METHODS \\ Subjects}

From a series of outpatients with relapsing-remitting MS diagnosed according to the McDonald criteria ${ }^{10,11}$ attending our Multiple Sclerosis Center, we selected 16 patients who had been diagnosed with OCD on the basis of the Diagnostic and Statistical Manual of Mental Disorders (4th edition). ${ }^{12}$ Written informed consent was obtained, after a description of the study, in line with the principles of the Declaration of Helsinki.

Inclusion criteria were age between 18 and 50 years, mild level of disability (ie, EDSS score below 3.5), ${ }^{13}$ and right-handedness. Exclusion criteria were any pre-existing history of obsessive-compulsive traits, tic disorder, or other OCD spectrum disorders; any first-degree relative with OCD; other concomitant neurologic or psychiatric diseases; cognitive impairment; a new relapse and/or systemic corticosteroid therapy in the 30 days before study entry; and major medical illnesses.

Fifteen patients with relapsing-remitting MS without any psychiatric disorders, satisfying the same inclusion and exclusion criteria and matched for age and clinical disability, were included as a CG.

All patients were on immunomodulatory therapy and underwent a neurologic evaluation, including administration of the EDSS, a psychiatric assessment, a cognitive assessment, and MR imaging.

\section{Psychiatric Assessment}

OCD diagnosis was based on the Diagnostic and Statistical Manual of Mental Disorders. The severity of the OCD was assessed by the means of the Y-BOCS. ${ }^{14}$ The Y-BOCS is a 10 -item clinician-administered scale developed to assess the severity of obsessions and compulsions, regardless of the number and type of obsessions or compulsions present. Obsessions and compulsions are rated according to the amount of resistance to them, distress over them, control over them, interference from them, and time spent on them. The following total scores are considered as a severity index for OCD symptoms: $0-7=$ subclinical; $8-15=$ mild; $16-23=$ moderate; $24-31=$ severe; $32-40=$ extreme.

All patients underwent a psychiatric interview to rule out any other psychiatric disorders. The Ham- $\mathrm{D}^{15}$ and Ham- $\mathrm{A}^{16}$ were used for rating depression and anxiety, respectively.

\section{Cognitive Assessment}

A battery of neuropsychologic tests was administered to each subject by a psychologist (E.M.Q.) who had more than 7 years of experience in neuropsychologic test design and measurement and who was blinded to the functional MR imaging results.

The neuropsychologic test battery included the Stroop Color Naming Test, ${ }^{17}$ Raven Progressive Matrices, ${ }^{18}$ Tower of Lon- don, ${ }^{19}$ Rey Complex Figure (copy and recall), ${ }^{20}$ Trail-Making Test $\mathrm{A}$ and $\mathrm{B},{ }^{21}$ Rey Auditory Verbal Learning, ${ }^{22}$ and Digit Span. ${ }^{23}$

\section{MR Imaging Acquisition}

MR images were acquired with a $1.5 \mathrm{~T}$ magnet (Gyroscan NT 15; Philips Medical Systems, Best, the Netherlands). All patients were investigated during a single session with the following sequences: 1) an axial dual-echo fast spin-echo sequence on 48 contiguous 3-mm-thick sections (TR: $2150 \mathrm{~ms}$, TE: 30/120 ms; echo-train length: 12 ; matrix: $256 \times 256$; field of view: $240 \mathrm{~mm}$ ); 2) a $3 \mathrm{D}$ T1-weighted fast-field echo sequence on 150 contiguous axial 1-mm-thick sections (TR: $40 \mathrm{~ms}$; TE: $4 \mathrm{~ms}$; flip angle: $30^{\circ}$; matrix: $512 \times 512$; field of view: $240 \mathrm{~mm}$ ); 3) a DTI with a single-shot echo-planar pulse sequence on 25 contiguous 5-mm-thick sections (TR: 3694 ms, TE: 95 ms; matrix: $128 \times 128$; field of view: $240 \mathrm{~mm}$ ) with diffusion gradients applied along 32 noncollinear directions using 2 b-values ( 0 and 1000 seconds $/ \mathrm{mm}^{2}$ ); and 4) an axial T1-weighted spin-echo sequence on 48 contiguous 3-mmthick sections (TR: $600 \mathrm{~ms}$, TE: $15 \mathrm{~ms}$; matrix: $256 \times 256$; field of view: $240 \mathrm{~mm}$ ) after injection of $0.2 \mathrm{mmol} / \mathrm{kg}$ of gadoliniumdiethylene-triamine pentaacetic acid.

\section{Image Analysis and Postprocessing}

Image data processing was performed on a personal computer running Jim 5.0 software (Xinapse System, Leicester, England), SPM5 software (Wellcome Department of Cognitive Neurology, London, England), the FSL 4.0 software package (FMRIB Image Analysis Group, Oxford, England; http://www.fmrib.ox.ac.uk/ fsl), and MATLAB 7.0 software (Mathworks, Natick, Massachusetts). We used Jim 5.0 for LV calculation, SPM5 for VBM analysis, and FSL for FA calculation and TBSS.

\section{Lesion Volume}

On conventional proton attenuation, the LV was calculated by Jim 5.0 software. The LV yielded the following data for every subject: a quantification of the lesion burden and a binary lesion mask needed for further image processing (both VBM and TBSS). Lesion masks were then transformed into standard space and averaged to yield a mean lesion mask across subjects. We also calculated a regional LV in the frontal and temporal lobes of each cerebral hemisphere on the basis of anatomic landmarks. ${ }^{24}$

\section{Voxel-Based Morphometry}

3D T1 images underwent automated segmentation to create GM, WM, and CSF images. The VBM protocol, which consisted of an iterative combination of segmentations and normalizations, yielded a GM probability map.

Normalized GM images were modulated, that is, multiplied by the local value derived from the deformation field, thereby preserving within-voxel volumes that might have been altered during nonlinear normalization. For all subjects, lesion masks were used to remove lesional tissue incorrectly classified as GM from the output of segmentation. GM, WM, and CSF volumes were recorded and used to calculate the intracranial volume as GM + $\mathrm{WM}+\mathrm{CSF}$. The brain parenchymal fraction was calculated as follows: $(\mathrm{GM}+\mathrm{WM}) /$ intracranial volume.

Data were smoothed using a $12-\mathrm{mm}$ full width at half maxi- 
Table 1: Demographic and clinical data in 16 patients with MS with OCD and in 15 patients with MS without psychiatric disorders (CG)

\begin{tabular}{lcc} 
& MS OCD & MS CG \\
\hline Age (yrs) & $39.5 \pm 8.0$ & $36.8 \pm 7.7$ \\
Gender (F/M) & $10 / 6$ & $10 / 5$ \\
EDSS & $2.0(1-3)$ & $1.5(1-2.5)$ \\
Disease duration (yrs) & $5.75 \pm 3.8$ & $5.87 \pm 4.1$ \\
Education (yrs) & $15.6 \pm 3.1$ & $14.8 \pm 2.8$ \\
\hline
\end{tabular}

Note:-All values are expressed as mean \pm standard deviation, except for gender (ratio) and EDSS (median and range). No statistically significant difference in any demographic and clinical variable was found between the 2 groups by unpaired $t$ test (except for gender).

mum Gaussian kernel. The processed images of GM in MS OCD and MS CG were compared through voxelwise comparisons.

Based on an a priori hypothesis, we restricted VBM analysis to voxels included in a mask, which was constructed on the automated anatomic labeling map, ${ }^{24}$ using MRIcro 1.38 software (http://www.sph.sc.edu/comd/rorden/mricro.html), by selecting regions found to be affected in primary OCD in previous studies; that is, the middle and inferior frontal gyri, orbitofrontal cortex, anterior cingulum, thalamus, basal ganglia, temporal pole, and superior, middle, and inferior temporal gyri and insula, bilaterally. ${ }^{25-28}$ To calculate regional GM volume differences between MS OCD and MS CG, we used a 2-sample $t$ test in SPM5, entering age, EDSS, LV and disease duration as nuisance variables. Statistical threshold was set at $P<.05$, false discovery rate corrected at the voxel level.

\section{TBSS and Mean FA}

DTI data were first corrected for eddy currents and involuntary motion using affine registration ${ }^{29}$; the diffusion tensor was estimated by multivariate linear regression, and FA maps were calculated. FA images were analyzed using TBSS, an FSL tool. ${ }^{30}$ This involves realigning all images to a common template, creating a "skeleton" by computing and thinning the mean FA image, and projecting each subject's FA onto the skeleton. These data were then fed into a voxelwise cross-subject statistical analysis, which, in our study, was performed to identify differences in the FA areas between groups of patients. TBSS was restricted to the normalappearing white matter by excluding from the analysis all voxels in which at least $10 \%$ of the patient population had a lesion (based on the mean T2 lesion mask). We performed the voxelwise analysis using an unpaired $t$ test with a permutation-based inference (2000 permutations) corrected for multiple comparisons, with a significance level of $P<.05$. The mean FA was also calculated for each subject.

\section{RESULTS}

There were no significant differences in demographic and clinical variables (age, sex, EDSS, disease duration, educational level) between patients with MS who developed OCD and patients with MS who did not (Table 1).

The mean Y-BOCS score in the OCD group was $26.13 \pm 6.4$. OCD severity was moderate in 6 patients, severe in 5 , and extreme in the remaining 5. No patient exhibited symptoms of anxiety or depression. No significant differences in either Ham-D (OCD =
Table 2: Neuropsychologic evaluation in 16 patients with MS with $O C D$ and in 15 patients with MS without psychiatric disorders (CG)

\begin{tabular}{lcc}
\hline \multicolumn{1}{c}{ Neuropsychologic Test } & MS OCD & MS CG \\
\hline Rey Auditory Verbal Learning & $34.1 \pm 7.9$ & $35.1 \pm 6.5$ \\
Rey Auditory Verbal Learning Recall & $10.2 \pm 2.7$ & $9.5 \pm 2.1$ \\
Trail-Making Test A-B & $40.7 \pm 18.5$ & $43.8 \pm 24.2$ \\
Raven Progressive Matrices & $29.7 \pm 3.4$ & $29.7 \pm 3.2$ \\
Tower of London & $26.2 \pm 6.6$ & $29.3 \pm 3.4$ \\
Rey Complex Figure-Copy & $33.5 \pm 2.3$ & $33.3 \pm 2.5$ \\
Rey Complex Figure-Recall & $18.4 \pm 3.9$ & $18.1 \pm 4.4$ \\
Digit Span & $5.6 \pm 0.7$ & $5.5 \pm 1.1$ \\
Stroop Color Naming Test & $55.8 \pm 9.6$ & $46.7 \pm 1.4$ \\
\hline
\end{tabular}

Note:-All values are expressed as mean \pm standard deviation. No statistically significant difference in any neuropsychological test was found between groups by unpaired $t$ test.

Table 3: Regional and global LV values in 16 patients with MS with $O C D$ and in 15 patients with MS without psychiatric disorders

(CG)

\begin{tabular}{lcccccc}
\hline & \multicolumn{2}{c}{ MS OCD } & & \multicolumn{2}{c}{ MS CG } & \\
\cline { 2 - 3 } & $\mathbf{m m}^{\mathbf{3}}$ & $\%$ & & $\mathbf{m m}^{3}$ & $\%$ & $\boldsymbol{P}$ \\
\hline Left frontal & $2593 \pm 4092$ & 27 & & $1192 \pm 2141$ & 24 & .25 \\
Right frontal & $2523 \pm 3482$ & 27 & & $847 \pm 1132$ & 17 & .86 \\
Left temporal & $598 \pm 955$ & 6 & & $285 \pm 385$ & 6 & .24 \\
Right temporal & $783 \pm 1021$ & 8 & & $447 \pm 532$ & 9 & .26 \\
Global & $9440 \pm 6980$ & - & $4880 \pm 5756$ & - & .21 \\
\hline
\end{tabular}

Note:- LV values are expressed as mean \pm standard deviation. No statistically sig nificant difference in LV was found in any region between groups by unpaired $t$ test.

$8.6 \pm 3.1 ; \mathrm{CG}=6.9 \pm 2.6)$ or in Ham-A $(\mathrm{OCD}=11.2 \pm 2.8$; $\mathrm{CG}=9.8 \pm 2.9)$ were found between groups.

The cognitive evaluation excluded the presence of cognitive impairment in patients in both groups and any significant difference in cognitive performance between the MS OCD group and the MS CG (Table 2).

\section{Lesion Volume}

Regional and global LVs are shown in Table 3. Although LV values were higher in patients with MS and OCD than in patients with MS without psychiatric disorders, there were no significant differences in either the global or regional LVs between the 2 groups. Moreover, the lesion distribution between the frontal and temporal lobes and between the right and the left hemispheres was comparable in the 2 groups.

\section{Voxel-Based Morphometry}

The global GM volume was slightly, though not significantly, lower in the OCD group than in the control group (530.2 \pm 70.6 versus $578.8 \pm 68.5)$. The global GM volume was not correlated with the Y-BOCS scores.

The VBM analysis revealed a set of clusters of reduced GM volume in patients with OCD that was located in the right inferior frontal gyrus $(Z=2.71$; coordinates: $48,14,-30)$, inferior temporal gyrus $(Z=3.30$, coordinates: $50,-24,-28)$, and middle temporal gyrus $(Z=2.90$, coordinates: $54,-4,-26)$ (2-sample $t$ test, $P<.05$, false discovery rate corrected at the voxel level) (Fig 1). No brain area showed greater GM volume in the MS OCD group than in the MS CG. 


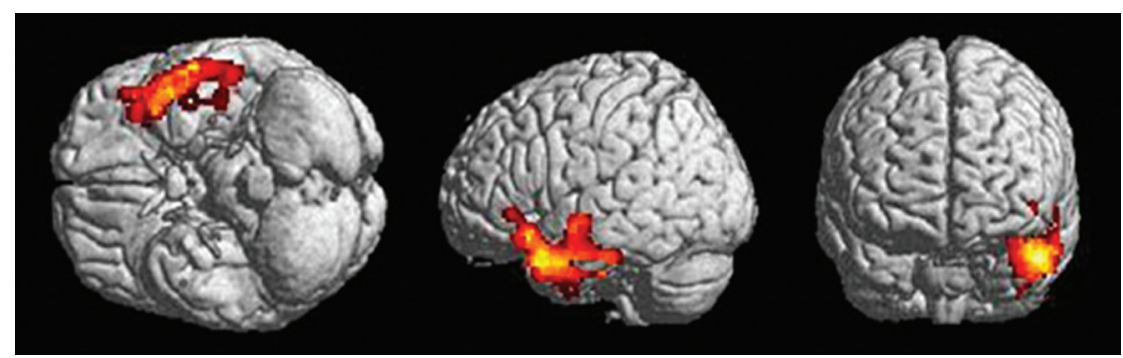

FIG 1. Images show focal decrease in GM volume in 16 patients with MS and OCD compared with 15 patients with MS without psychiatric disorders (CG). Clusters of decreased GM volume (red/yellow) are overlapped with a rendered brain. Clusters that are significant at $P<.05$ (2-sample $t$ test; false discovery rate corrected) are observed in the right inferior frontal gyrus ( $Z=2.71$; coordinates: $48,14,-30)$, inferior temporal gyrus $(Z=3.30$, coordinates: $50,-24,-28)$ and middle temporal gyrus $(Z=2.90$, coordinates: $54,-4,-26)$.

\section{TBSS and Mean FA}

There was no significant difference between the 2 patient groups in the mean FA values (FA in OCD: $0.43 \pm 0.03$; FA in CG: $0.45 \pm$ 0.02 ). Voxelwise analysis by TBSS did not reveal any area of significant between-group difference in FA in normal-appearing WM. The correlation between the FA and Y-BOCS scores in the OCD group was not statistically significant.

\section{DISCUSSION}

When we compared patients with MS and OCD and those without psychiatric disorders, we found a significant reduction in GM volume in the frontotemporal cortex in patients with OCD, affecting in particular the inferior frontal gyrus and the inferior and middle temporal gyri on the right side.

As the neurobiologic mechanisms underlying psychiatric symptoms in MS have seldom been investigated, these remain obscure. Although neuroimaging techniques have been repeatedly used to investigate primary $\mathrm{OCD},,^{7-9,31,32}$ no neuroimaging study has systematically addressed the occurrence of OCD during the course of MS. In a single case, the development of obsessivecompulsive symptoms after the diagnosis of MS was found to be associated with the emergence of a right parietal white matter multiple sclerosis plaque. ${ }^{33}$

In primary $\mathrm{OCD}$, morphometric alterations have been consistently found not only in the frontal lobe (dorsolateral, orbitofrontal, and anterior cingulate cortices) and the basal ganglia ${ }^{34,35}$ but also in the supramarginal gyrus, ${ }^{36}$ anterior superior temporal cortex, ${ }^{25}$ hippocampus, and amygdala. ${ }^{31}$

Some evidence of the role of the temporal lobe in the pathophysiology of OCD has been yielded by studies on OCD associated with neurologic disorders, particularly with epilepsy. The fact that obsessive-compulsive symptoms may be present in over $20 \%$ of patients with drug-resistant temporal lobe epilepsy suggests that temporal lobe epilepsy and OCD may share some neuroanatomic circuit dysfunctions. ${ }^{37,38}$ Obsessive-compulsive symptoms may be more severe in patients with right-sided epilepsy than in those with left-sided epilepsy. ${ }^{37,39}$ OCD may also occur after temporal lobe resection performed as treatment for drug-resistant epilepsy. ${ }^{40,41}$

Secondary obsessive-compulsive symptoms have also been reported after encephalitis or traumatic brain injury ${ }^{42,43}$; decreased blood flow in the temporal lobes, as well as cortical perfusion abnormalities in the frontal lobes, have been described in OCD, acquired as a result of various neurologic conditions. ${ }^{44}$

Apart from the focal volume decrease in the right frontotemporal cortex detected in the patients with MS and OCD, we did not find any other abnormalities in the global or regional FA, which is an index of white matter tract integrity, nor did we observe more macroscopic white matter lesions in the right hemisphere. This finding is in contrast to the results of recent DTI studies in primary OCD, which have reported abnormalities in the corpus callosum and subcortical frontal white matter associated with either increased ${ }^{32}$ or decreased ${ }^{45}$ structural connectivity. Differences in patient characteristics and/or in methods of MR acquisition and data analysis could account for this discrepancy.

\section{CONCLUSIONS}

Our study indicates that OCD associated with MS has an anatomic substrate and may be related to damage in the right frontotemporal cortex. Further studies are warranted to shed light on the mechanisms underlying the association between frontotemporal damage and the development of OCD in MS, and to investigate whether there is a cause-effect relationship between these.

Disclosures: Carlo Pozzilli_UNRELATED: Board Membership: Advisory Board for Merck Serono, Biogen, Sanofi Aventis, Teva, Bayer; Grants/Grants Pending: Merck Serono, * Biogen, ${ }^{*}$ Bayer;, Payment for Lectures (including service on speakers bureaus): Biogen, Merck Serono, Bayer, Sanofi Aventis, Novartis, Teva; Payment for Manuscript Preparation: Bayer; Payment for Development of Educational Presentations: Serono Symposia; Travel/Accommodations/Meeting Expenses Unrelated to Activities Listed: Biogen, Bayer, Novartis, Merck Serono, Teva, Sanofi Aventis. (*Money paid to institution)

\section{REFERENCES}

1. Wilken JA, Sullivan C. Recognizing and treating common psychiatric disorders in multiple sclerosis. Neurologist 2007;13:343-54

2. Papathanasopoulos PG, Papadimitriou GN. Psychiatric aspects of multiple sclerosis. Int Rev Psychiatry 2010;22:1

3. Feinstein A. Multiple sclerosis and depression. Mult Scler 2011;17:1276-81

4. Patten SB, Svenson LW, Metz LM. Psychotic disorders in MS: population-based evidence of an association. Neurology 2005;65: 1123-25

5. Kessler RC, McGonagle KA, Zhao S, et al. Lifetime and 12-month prevalence of DSM-III-R psychiatric disorders in the United States. Results from the National Comorbidity Survey. Arch Gen Psychiatry 1994;51:8-19

6. Korostil M, Feinstein A. Anxiety disorders and their clinical correlates in multiple sclerosis patients. Mult Scler 2007;13:67-72

7. Menzies L, Chamberlain SR, Laird AR, et al. Integrating evidence from neuroimaging and neuropsychological studies of obsessivecompulsive disorder: the orbitofronto-striatal model revisited. Neurosci Biobehav Rev 2008;32:525-49

8. Harrison BJ, Soriano-Mas C, Pujol J, et al. Altered corticostriatal functional connectivity in obsessive-compulsive disorder. Arch Gen Psychiatry 2009;66:1189-200

9. Radua J, van den Heuvel OA, Surguladze S, et al. Meta-analytical comparison of voxel-based morphometry studies in obsessive- 
compulsive disorder vs other anxiety disorders. Arch Gen Psychiatry 2010;67:701-11

10. McDonald WI, Compston A, Edan G, et al. Recommended diagnostic criteria for multiple sclerosis: guidelines from the International Panel on the Diagnosis of Multiple Sclerosis. Ann Neurol 2001;50:121-27

11. Polman CH, Reingold SC, Banwell B, et al. Diagnostic criteria for multiple sclerosis: 2010 revisions to the McDonald criteria. Ann Neurol 2011;69:292-302

12. American Psychiatric Association. Diagnostic and Statistical Manual of Mental Disorders (4th ed., text revision). Washington, DC: American Psychiatric Association; 2000

13. Kurtzke JF. Rating neurologic impairment in multiple sclerosis: an expanded disability status scale (EDSS). Neurology 1983;33:1444-52

14. Goodman WK, Price LH, Rasmussen SA, et al. The Yale-Brown Obsessive Compulsive Scale. I. Development, use, and reliability. Arch Gen Psychiatry 1989;46:1006-11

15. Hamilton M. Hamilton Scale for Depression. J Neurol Neurosurg Psychiatry 1960;23:56-62

16. Hamilton $\mathrm{M}$. The assessment of anxiety states by rating. $\mathrm{Br} J \mathrm{Med}$ Psychol 1959;32:50-55

17. Stroop J. Studies of interference in serial verbal reactions. Princeton, New Jersey: Princeton University Press; 1935/2004

18. Raven J, Raven JC, Court JH. Manual for Raven's Progressive Matrices and Vocabulary Scales. San Antonio, Texas: Harcourt Assessment; 2003

19. Morris RG, Ahmed S, Syed GM, et al. Neural correlates of planning ability: frontal lobe activation during the Tower of London test. Neuropsychologia 1993;31:1367-78

20. Meyers J, Meyers K. Rey Complex Figure Test and Recognition Trial: Professional Manual. Odessa, FL: Psychological Assessment Resources; 1995

21. Reitan R. Trail-Making Test: Manual for Administration and Scoring. Tucson, Arizona: Reitan Neuropsychology Laboratory; 1992

22. Rey A. Mémorisation d'une série de 15 mots en 5 répétitions. Paris, France: Presses Universitaires des France; 1958

23. Wechsler D. Wechsler Adult Intelligence Scale III. New York, New York: The Psychological Corporation; 1997

24. Tzourio-Mazoyer N, Landeau B, Papathanassiou D, et al. Automated anatomical labeling of activations in SPM using a macroscopic anatomical parcellation of the MNI MRI single-subject brain. NeuroImage 2002;15:273-89

25. Choi J-S, Kim H-S, Yoo SY, et al. Morphometric alterations of anterior superior temporal cortex in obsessive-compulsive disorder. Depress Anxiety 2006;23:290-96

26. Yoo SY, Roh M-S, Choi J-S, et al. Voxel-based morphometry study of gray matter abnormalities in obsessive-compulsive disorder. $J$ Korean Med Sci 2008;23:24-30

27. Rotge J-Y, Langbour N, Guehl D, et al. Gray matter alterations in obsessive-compulsive disorder: an anatomic likelihood estimation meta-analysis. Neuropsychopharmacology 2010;35:686-91

28. Nishida S, Narumoto J, Sakai Y, et al. Anterior insular volume is larger in patients with obsessive-compulsive disorder. Prog Neuropsychopharmacol Biol Psychiatry 2011;35:997-1001

29. Jenkinson M, Smith S. A global optimisation method for robust affine registration of brain images. Med Image Anal 2001;5:143-56

30. Smith SM, Jenkinson M, Johansen-Berg H, et al. Tract-based spatial statistics: voxelwise analysis of multi-subject diffusion data. NeuroImage 2006;31:1487-505

31. Atmaca M, Yildirim $\mathrm{H}$, Ozdemir $\mathrm{H}$, et al. Hippocampus and amygdalar volumes in patients with refractory obsessive-compulsive disorder. Prog Neuropsychopharmacol Biol Psychiatry 2008;32: 1283-86

32. Li F, Huang X, Yang Y, et al. Microstructural brain abnormalities in patients with obsessive-compulsive disorder: diffusion-tensor MR imaging study at 3.0 T. Radiology 2011;260:216-23

33. Douzenis A, Michalopoulou PG, Voumvourakis C, et al. Obsessivecompulsive disorder associated with parietal white matter multiple sclerosis plaques. World J Biol Psychiatry 2009;10:956-60

34. Rotge J-Y, Guehl D, Dilharreguy B, et al. Meta-analysis of brain volume changes in obsessive-compulsive disorder. Biol Psychiatry 2009;65:75-83

35. Rotge J-Y, Langbour N, Jaafari N, et al. Anatomical alterations and symptom-related functional activity in obsessive-compulsive disorder are correlated in the lateral orbitofrontal cortex. Biol Psychiatry 2010;67:e37-38

36. Koprivová J, Horácek J, Tintera J, et al. Medial frontal and dorsal cortical morphometric abnormalities are related to obsessive-compulsive disorder. Neurosci Lett 2009;464:62-66

37. Isaacs KL, Philbeck JW, Barr WB, et al. Obsessive-compulsive symptoms in patients with temporal lobe epilepsy. Epilepsy Behav 2004;5:569-74

38. Kaplan PW. Obsessive-compulsive disorder in chronic epilepsy. Epilepsy Behav 2011;22:428-32

39. Sperli F, Rentsch D, Despland PA, et al. Psychiatric comorbidity in patients evaluated for chronic epilepsy: a differential role of the right hemisphere? Eur Neurol 2009;61:350-57

40. Kulaksizoglu IB, Bebek N, Baykan B, et al. Obsessive-compulsive disorder after epilepsy surgery. Epilepsy Behav 2004;5:113-18

41. Roth RM, Jobst BC, Thadani VM, et al. New-onset obsessive-compulsive disorder following neurosurgery for medication-refractory seizure disorder. Epilepsy Behav 2009;14:677-80

42. Pewter SM, Williams WH, Haslam C, et al. Neuropsychological and psychiatric profiles in acute encephalitis in adults. Neuropsychol Rehabil 2007; 17:478-505

43. Grados MA, Vasa RA, Riddle MA, et al. New onset obsessive-compulsive symptoms in children and adolescents with severe traumatic brain injury. Depress Anxiety 2008;25:398-407

44. Hugo F, van Heerden B, Zungu-Dirwayi N, et al. Functional brain imaging in obsessive-compulsive disorder secondary to neurological lesions. Depress Anxiety 1999;10:129-36

45. Oh JS, Jang JH, Jung WH, et al. Reduced fronto-callosal fiber integrity in unmedicated OCD patients: a diffusion tractography study. Hum Brain Map 2012;33:2441-52 